\title{
Deficiência de micronutrientes e crescimento linear: revisão sistemática de estudos observacionais
}

\author{
Micronutrient deficiencies and linear growth: \\ a systematic review of observational studies
}

Dixis Figueroa Pedraza ${ }^{1}$

Ana Carolina Dantas Rocha ${ }^{1}$

Márcia Cristina Sales ${ }^{1}$

${ }^{1}$ Programa de PósGraduação em Saúde Pública, Pró-Reitoria de Pós-Graduação e Pesquisa (PRPGP), Universidade Estadual da Paraíba. Av. das Baraúnas 351 - Campus Universitário, Bodocongó. 58.109-753 Campina Grande PB Brasil. dixisfigueroa@gmail.com
Abstract This article seeks to evaluate the association of iron, vitamin $A$ and zinc deficiencies with linear growth retardation. A systematic review of electronic databases in PubMed, LILACS and SciELO was conducted. Scientific papers published between January 1995 and March 2010 were selected, inserting the key words: (growth OR nutritional status) AND (child, preschool OR infant) AND (zinc AND iron AND vitamin A) OR (zinc AND iron) OR (zinc AND vitamin A) OR (iron AND vitamin A). Fourteen observational design studies were reviewed. In the cohort studies (two), one indicated a statistical association between iron levels and stunting; and the other revealed a statistical association between serum ferritin concentrations and an increase in height. Ten cross-sectional studies investigated the statistical association between micronutrient deficiencies and stunting, three of which resulted in an association with iron, two with vitamin $A$ and none with zinc. Elucidation of the association between stunting and iron, vitamin $A$ and zinc deficiencies involves difficulties of a biological nature and also related to the magnitude of these deficiencies, indicating the importance of a methodological standardization of the studies.

Key words Linear growth, Height, Stunting, Iron, Vitamin A, Zinc
Resumo O objetivo do artigo é avaliar a associação das deficiências de ferro, vitamina $A$ e zinco com o déficit de crescimento linear. Revisão sistemática nas bases de dados eletrônicas PubMed, LILACS e SciELO. Foram selecionados artigos publicados entre janeiro de 1995 e março de 2010, considerando os descritores: (growth OR nutritional status) AND (child, preschool OR infant) AND (zinc AND iron AND vitamin A) OR (zinc AND iron) OR (zinc AND vitamin A) OR (iron AND vitamin $A)$. Foram revisados 14 estudos de delineamento observacional. Dos estudos tipo coorte (dois), um indicou associação estatística entre o estado nutricional de ferro e o déficit de estatura; $o$ outro apontou associação estatística entre as concentrações de ferritina sérica e o ganho de estatura. Dez estudos transversais investigaram a associação estatística entre as deficiências de micronutrientes e o déficit de estatura, resultando três deles na associação para o ferro, dois para a vitamina A e nenhum para o zinco. O esclarecimento sobre a associação entre o déficit de estatura e as deficiências de ferro, vitamina A e zinco dificultase por fatores de caráter biológico e relacionados à magnitude das deficiências, o que sugere a importância da padronização metodológica dos estudos. Palavras-chave Crescimento linear, Estatura, Déficit de estatura, Ferro, Vitamina A, Zinco 


\section{Introdução}

A deficiência de micronutrientes está relacionada com uma série de efeitos deletérios na infância, com consequente aumento das taxas de morbimortalidade, dentre outros agravos à saúde. Por isso, esta etapa da vida representa um momento biológico que merece o máximo de atenção com relação à oferta de micronutrientes, em especial ferro, vitamina A e zinco, haja vista sua importância no baixo peso ao nascer e no déficit de estatura $^{1,2}$.

Embora as deficiências de micronutrientes possam ocorrer isoladamente, elas usualmente existem de forma combinada, devendo-se, portanto, dar maior atenção às interações metabólicas que ocorrem entre elas. É reconhecida a interação entre o metabolismo do ferro, da vitamina A e do zinco, pois a deficiência de um destes nutrientes pode prejudicar a utilização dos demais pelo organismo humano. O zinco é requerido para a síntese hepática e para a secreção da proteína transportadora da vitamina A. A deficiência de ferro influencia os níveis séricos de retinol, pois a sua carência compromete o funcionamento normal da mucosa intestinal, dificultando a absorção da vitamina A. Em contrapartida, há uma correlação entre a Deficiência de Vitamina A (DVA) e a anemia, visto que a suplementação desta vitamina aumenta a mobilização hepática de ferro, estimulando a eritropoiese. Com relação à interação entre zinco e ferro, postula-se que altas concentrações de ferro podem afetar negativamente a absorção de zinco, efeito dependente da quantidade total de espécies iônicas, da dose de ferro, da relação molar Zn:Fe e da via de administração. Vários estudos têm mostrado que o efeito está presente principalmente quando esses minerais são administrados em solução, não sendo o mesmo observado no caso desses minerais serem ingeridos na dieta ${ }^{3-5}$.

Em geral, as deficiências de micronutrientes são consideradas fatores de risco potenciais do déficit de crescimento linear em crianças, sendo o ferro, a vitamina A e o zinco os de maior impacto no problema. Considera-se que as deficiências de ferro e vitamina A afetam o crescimento somente em condições de deficiência severa $(\mathrm{Hb}<$ $9,5 \mathrm{~g} / \mathrm{dL}$, retinol sérico $<0,35 \mu \mathrm{mol} / \mathrm{L}$, respectivamente), enquanto que até mesmo a deficiência leve de zinco pode ter consequências negativas no crescimento ${ }^{6,7}$.

Acredita-se que a maioria dos casos de déficit de estatura, resultado da interação entre o potencial genético e a biodisponibilidade de macro e micronutrientes durante o período de crescimento, esteja associada com a deficiência concomitante de vários micronutrientes ${ }^{2,8,9}$. As lacunas sobre a melhor estratégia de suplementação com micronutrientes para a prevenção e a redução do déficit de estatura de crianças nos países em desenvolvimento explicam a importância de um melhor conhecimento a esse respeito.

Com base no supracitado, o presente estudo tem por objetivo apresentar uma análise sumária de estudos a respeito da associação das deficiências de ferro, vitamina A e zinco com o déficit de crescimento linear, destacando aqueles que analisam minimamente dois micronutrientes.

\section{Metodologia}

O estudo é uma revisão sistemática de artigos científicos sobre deficiência múltipla de micronutrientes e déficit de estatura. Apresentam-se os resultados de estudos observacionais que analisam a associação entre o estado nutricional de dois ou mais dos micronutrientes ferro, vitamina A e zinco e o retardo de crescimento linear de crianças pré-escolares.

Os estudos considerados elegíveis foram identificados combinando os resultados de buscas, completadas em 9 de março de 2010, nas bases de dados PubMed (National Library of Medicine, Bethesda, MD), LILACS (Literatura Latinoamericana e do Caribe em Ciências da Saúde) e SciELO (Scientific Electronic Library Online).

A busca de artigos nas bases LILACS e SCIELO considerou a seguinte estratégia: todos os artigos contendo a combinação dos descritores (growth OR nutritional status) AND (child, preschool OR infant) AND (zinc AND iron AND vitamin A) OR (zinc AND iron) OR (zinc AND vitamin A) OR (iron AND vitamin A) em qualquer campo. A busca também considerou os correspondentes desses descritores nos idiomas inglês e espanhol.

Para a busca de artigos na base de dados MEDLINE foram utilizados os descritores anteriores considerando uma sequência ordenada de passos. Em um primeiro momento, foram identificados todos os artigos, independente do desenho de estudo. Em seguida, foram identificados os artigos do tipo ensaio clínico randomizado controlado (limitando a busca para esta opção). $\mathrm{Na}$ sequência, foram identificados os possíveis artigos do tipo observacional, considerando os artigos identificados no primeiro momento e não identificados no segundo. 
Em todos os casos a busca limitou-se a estudos nos idiomas inglês, espanhol ou português publicados entre 01/01/1995 e o dia da busca. Para o cômputo do total de estudos identificados, foi verificada a duplicação ou triplicação dos mesmos entre as diferentes bases de dados, sendo cada artigo contabilizado somente uma vez.

Com o objetivo de localizar artigos que não tivessem sido encontrados nas bases de dados, as listas de referências dos estudos incluídos foram utilizadas.

A partir dos estudos identificados, foram selecionados aqueles que parecessem preencher os critérios para sua inclusão considerando a leitura dos títulos e resumos pelos revisores. Todos os artigos selecionados foram avaliados pelos revisores considerando a leitura e a análise criteriosa do texto completo. Após esta ação, os artigos foram classificados como excluídos e incluídos considerando os critérios estabelecidos para esses fins (Quadro 1). Os artigos identificados a partir das listas de referências também respeitaram ditos critérios para sua inclusão.
Inicialmente foram identificados 190 artigos. Após análise dos títulos e resumos, foram selecionados 67 artigos que aparentemente preenchiam os critérios de inclusão. Com a leitura dos artigos na sua íntegra, foram incluídos outros nove que adequadamente preenchiam os critérios de inclusão. A utilização das listas de referências bibliográficas dos artigos incluídos possibilitou a identificação de mais cinco com os critérios pré-estabelecidos. O fluxo do número de estudos incluídos encontra-se na Tabela 1.

As informações selecionadas nos artigos para sua caracterização foram: autor e ano de publicação, país de realização do estudo, tipo de estudo, população e tamanho da amostra, variáveis de estudo, principais resultados do estudo e escore de qualidade.

A qualidade dos estudos foi avaliada através de checklist (lista de pontos) preparado pelos pesquisadores. O checklist consistiu de uma lista adaptada dos critérios de Downs e Black ${ }^{10}$ excluindo os itens relacionados apenas a estudos de intervenção. Para a construção do instrumento, foi consi-

Quadro 1. Critérios para a inclusão e exclusão dos artigos na revisão sistemática sobre deficiência de micronutrientes e crescimento linear de crianças pré-escolares (artigos que analisam minimamente dois micronutrientes).

\begin{tabular}{|c|c|c|}
\hline Parâmetros & Critérios de inclusão & Critérios de exclusão \\
\hline $\begin{array}{l}\text { Tipos de } \\
\text { estudos }\end{array}$ & $\begin{array}{l}\text { - estudos observacionais relacionados } \\
\text { com o tema da revisão }\end{array}$ & $\begin{array}{l}\text { - estudos que claramente não estão } \\
\text { relacionados com o tema } \\
\text { - estudos em animais } \\
\text { - estudos de intervenção* } \\
\text { - artigos de revisão } \\
\text { - livros ou tese }\end{array}$ \\
\hline Participantes & $\begin{array}{l}\text { - crianças } \\
\text { - amostra representativa e selecionada } \\
\text { aleatoriamente }\end{array}$ & $\begin{array}{l}\text { - idade inapropriada (adolescentes, } \\
\text { adultos, mulheres grávidas) } \\
\text { - crianças com patologias que podem } \\
\text { afetar o crescimento } \\
\text { - amostra não representativa e/ou não } \\
\text { selecionada aleatoriamente }\end{array}$ \\
\hline Exposição & $\begin{array}{l}\text { - indicadores bioquímicos de no } \\
\text { mínimo dois dos seguintes } \\
\text { micronutrientes: zinco, ferro, } \\
\text { vitamina A }\end{array}$ & $\begin{array}{l}\text { - estudos de avaliação de consumo de } \\
\text { alimentos ou de avaliação nutricional } \\
\text { de alimentos } \\
\text { - indicadores bioquímicos de um único } \\
\text { micronutriente }\end{array}$ \\
\hline Desfechos & $\begin{array}{l}\text { - crescimento: Z-escores de estatura } \\
\text { para idade, ganho de estatura }\end{array}$ & $\begin{array}{l}\text { - associação entre crescimento e } \\
\text { estado nutricional de micronutrientes } \\
\text { não avaliada** }\end{array}$ \\
\hline
\end{tabular}

* Considerou-se estudos de intervenção aqueles com suplementação e fortificação, assim como os estudos de avaliação do estado nutricional de crianças beneficiárias de algum programa ou política de alimentação e nutrição. ${ }^{* *}$ Considerou-se como associação os resultados de inferência estatística e a coexistência do déficit de estatura com as deficiências de micronutrientes. 


\begin{tabular}{|c|c|c|c|}
\hline 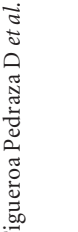 & $\begin{array}{l}\text { Tabela } 1 . \\
\text { crescimen }\end{array}$ & $\begin{array}{l}\text { studos excluídos e incluídos na revisão sistemática sobre deficiência de mic } \\
\text { o linear de crianças pré-escolares (artigos que analisam minimamente dois }\end{array}$ & $\begin{array}{l}\text { ronutrientes e } \\
\text { micronutrientes). }\end{array}$ \\
\hline & Etapas & Critérios de exclusão e inclusão & Número de artigos \\
\hline & 1 & Artigos identificados (bases de dados) & \\
\hline & & MEDLINE & 134 \\
\hline & & LILACS & 56 \\
\hline & & SCIELO & 10 \\
\hline & & Estudos em duplicata & 8 \\
\hline & & Estudos em triplicata & 2 \\
\hline & & Total de estudos identificados & 190 \\
\hline & 2 & Documentos e artigos excluídos & \\
\hline & & - estudos claramente não relacionados com o tema* & 21 \\
\hline & & - estudos em animais ${ }^{*}$ & 2 \\
\hline & & - estudos de intervenção* ${ }^{*}$ & 20 \\
\hline & & - artigos de revisão* & 13 \\
\hline & & - livros ou tese ${ }^{*}$ & 7 \\
\hline & & - idade inapropriada* & 26 \\
\hline & & - crianças com algum tipo de patologia ${ }^{* *}$ & 23 \\
\hline & & - amostra não representativa e/ou não selecionada aleatoriamente ${ }^{* *}$ & 4 \\
\hline & & $\begin{array}{l}\text { - avaliação de consumo de alimentos ou avaliação nutricional de } \\
\text { alimentos* }\end{array}$ & 17 \\
\hline & & - indicadores bioquímicos de um único micronutriente ${ }^{*}$ & 17 \\
\hline & & $\begin{array}{l}\text { - associação entre crescimento e estado nutricional de micronutrientes } \\
\text { não avaliada** }\end{array}$ & 31 \\
\hline & & Total de estudos excluídos & 181 \\
\hline & 3 & Estudos selecionados (leitura de títulos e resumos) & 67 \\
\hline & 4 & Estudos incluídos (leitura na íntegra dos artigos) & 9 \\
\hline & 5 & Estudos identificados e incluídos segundo listas de referências & 5 \\
\hline & 6 & Total de estudos incluídos & 14 \\
\hline
\end{tabular}

* Leitura de títulos e resumos. ${ }^{* *}$ Leitura na íntegra dos artigos.

derada a Declaração STROBE ${ }^{11}$ para a comunicação de estudos observacionais e as seguintes diretrizes para a elaboração de listas de avaliação da qualidade destes estudos ${ }^{12}$ : i) o instrumento deve ser de fácil utilização, ter um número pequeno de itens e ser do tipo checklist (evitar a medição em forma de escala); ii) o instrumento não deve deixar de considerar como foi realizada a seleção dos participantes, a medição das variáveis e o controle das variáveis de confusão, assim como o potencial do estudo/desenho para o controle dos vieses associados aos aspectos anteriores.

Sendo assim, analisaram-se os artigos com base na: (1) qualidade da descrição dos objetivos; (2) qualidade da descrição do desfecho de estudo; (3) qualidade da caracterização da amostra (descrição dos participantes e dos critérios de elegibilidade); (4) qualidade da descrição e discussão dos principais fatores de confusão; (5) qualidade da descrição das perdas de participantes; (6) qualidade da descrição dos principais resultados do estudo; (7) comprovação da representatividade da amostra estudada em relação à população de estudo; (8) descrição do cálculo da amostra e do processo de amostragem; (9) acurácia dos instrumentos utilizados para medir o desfecho; (10) apropriação dos testes estatísticos às características das variáveis; (11) avaliação correta dos grupos de comparação (iguais períodos de seguimento para os estudos de coorte, iguais períodos de tempo entre a exposição e o desfecho para os estudos caso controle); (12) adequação dos grupos de comparação (recrutados da mesma população e no mesmo período de tempo); (13) adequação do ajuste para os principais fatores de confusão ou apropriação dos testes estatísticos utilizados para seu controle. O escore de qualidade de cada artigo correspondeu à soma do total de itens avaliados como positivos.

\section{Resultados}

Foram incluídos $14 \operatorname{artigos}^{13-26}$, sendo 12 estudos transversais ${ }^{13-24}$ e dois estudos de coorte ${ }^{25,26}$, os quais estão resumidos na Tabela 2 . 
Tabela 2. Estudos observacionais sobre deficiência de micronutrientes e crescimento linear de crianças pré-escolares (artigos que analisam minimamente dois micronutrientes).

\begin{tabular}{|c|c|c|c|c|c|c|c|}
\hline \multirow[t]{2}{*}{ Autor, ano } & \multirow[t]{2}{*}{ País } & \multirow{2}{*}{$\begin{array}{l}\text { Tipo de } \\
\text { estudo }\end{array}$} & \multirow{2}{*}{$\begin{array}{c}\text { População } \\
\text { (amostra) }\end{array}$} & \multicolumn{2}{|c|}{ Dados de interesse } & \multirow[t]{2}{*}{ Principais resultados ${ }^{*}$} & \multirow[t]{2}{*}{ Escore $^{* *}$} \\
\hline & & & & Exposição & Desfecho & & \\
\hline $\begin{array}{l}\text { Chen et al., } \\
2009^{(13)}\end{array}$ & China & Transversal & $\begin{array}{c}\text { Crianças de } \\
2-7 \text { anos } \\
(459)\end{array}$ & $\begin{array}{c}\text { Ferro } \\
\text { Vitamina A }\end{array}$ & $\begin{array}{c}\text { Z-escore } \\
\text { de E/I } \\
\text { Z-escore } \\
\text { de } P / E \\
\text { Z-escore } \\
\text { de } P / I\end{array}$ & $\begin{array}{l}\text { - E/I<2Z: } 6,3 \% \\
\text { - P/E<2Z: } 3,9 \% \\
\text { - P/I<2Z: } 1,5 \% \\
\text { - Anemia: } 79,0 \% \\
\text { - Deficiência de vitamina A: } \\
6,3 \% \\
\text { - Ausência de associação } \\
\text { estatística entre a anemia e os } \\
\text { déficits antropométricos } \\
\text { - Associação estatística entre as } \\
\text { concentrações de retinol sérico e } \\
\text { de hemoglobina } \\
\text { - Coexistência dos déficits E/I, } \\
\text { P/E e P/I com a anemia em } \\
24,1 \% ; 14,3 \% \text {; e } 16,7 \% \text { das } \\
\text { crianças, respectivamente } \\
\text { - Coexistência da deficiência de } \\
\text { vitamina A com a anemia em } \\
41,4 \% \text { das crianças }\end{array}$ & 12 \\
\hline $\begin{array}{l}\text { Lander et al., } \\
2008^{(14)}\end{array}$ & Mongólia & Transversal & $\begin{array}{c}\text { Crianças de } \\
\text { 6-36 meses } \\
(243)\end{array}$ & $\begin{array}{c}\text { Ferro } \\
\text { Vitamina A } \\
\text { Zinco }\end{array}$ & $\begin{array}{c}\text { Z-escore } \\
\text { de E/I } \\
\text { Z-escore } \\
\text { de } P / E \\
\text { Z-escore } \\
\text { de } P / I\end{array}$ & $\begin{array}{l}\text { - E/I<2Z: } 14,5 \% \\
\text { - P/E<Z: } 9,0 \% \\
\text { - PI<2Z: } 4,0 \% \\
\text { - Anemia: } 24,0 \% \\
\text { - Deficiência de vitamina A: } \\
\text { 33,0\% } \\
\text { - Deficiência de zinco: } 74,0 \% \\
\text { - Ausência de associação } \\
\text { estatística entre a deficiência de } \\
\text { zinco e o déficit de estatura } \\
\text { - Ausência de correlação entre as } \\
\text { concentrações de retinol sérico e } \\
\text { de hemoglobina } \\
\text { - Ausência de correlação entre as } \\
\text { concentrações de retinol sérico e } \\
\text { de zinco sérico } \\
\text { - Coexistência da deficiência de } \\
\text { dois ou mais micronutrientes } \\
\text { em } 78,0 \% \text { das crianças }\end{array}$ & 10 \\
\hline $\begin{array}{l}\text { Del Real et } \\
\text { al., } 2007^{(15)}\end{array}$ & Venezuela & Transversal & $\begin{array}{c}\text { Crianças de } \\
\text { 4-7 anos (151) }\end{array}$ & $\begin{array}{c}\text { Ferro } \\
\text { Vitamina A }\end{array}$ & $\begin{array}{c}\text { Z-escore } \\
\text { de E/I } \\
\text { Z-escore } \\
\text { de } P / E\end{array}$ & $\begin{array}{l}\text { - E/I<2Z: } 7,0 \% \\
\text { - P/E<2Z: } 2,3 \% \\
\text { - Anemia: } 25,9 \% \\
\text { - Deficiência de vitamina A: } \\
0,0 \% \\
\text { - Associação estatística entre a } \\
\text { anemia e o déficit de estatura } \\
\text { - Associação estatística entre as } \\
\text { concentrações de retinol sérico e } \\
\text { de hemoglobina }\end{array}$ & 07 \\
\hline
\end{tabular}


Tabela 2. continuação

\begin{tabular}{|c|c|c|c|c|c|c|c|}
\hline \multirow[t]{2}{*}{ Autor, ano } & \multirow[t]{2}{*}{ País } & \multirow{2}{*}{$\begin{array}{l}\text { Tipo de } \\
\text { estudo }\end{array}$} & \multirow{2}{*}{$\begin{array}{c}\text { População } \\
\text { (amostra) }\end{array}$} & \multicolumn{2}{|c|}{ Dados de interesse } & \multirow[t]{2}{*}{ Principais resultados ${ }^{*}$} & \multirow[t]{2}{*}{ Escore $^{* *}$} \\
\hline & & & & Exposição & Desfecho & & \\
\hline $\begin{array}{l}\text { Mamiro et } \\
\text { al., } 2005^{(19)}\end{array}$ & Tanzânia & Transversal & $\begin{array}{c}\text { Crianças de } \\
3-23 \text { meses } \\
(378)\end{array}$ & $\begin{array}{l}\text { Ferro } \\
\text { Zinco }\end{array}$ & $\begin{array}{c}\text { Z-escore } \\
\text { de } E / I \\
\text { Z-escore } \\
\text { de } P / E\end{array}$ & $\begin{array}{l}\text { - E/I<2Z: } 35,0 \% \\
\text { - P/E<2Z: } 1,3 \% \\
\text { - Anemia: } 79,0 \% \\
\text { - Deficiência de zinco (dado não } \\
\text { disponível) } \\
\text { - Ausência de associação } \\
\text { estatística entre as } \\
\text { concentrações de } \\
\text { micronutrientes e os índices } \\
\text { antropométricos } \\
\text { - O peso ao nascer e o IMC } \\
\text { materno foram os principais } \\
\text { preditores da estatura infantil }\end{array}$ & 11 \\
\hline $\begin{array}{l}\text { Castejon et } \\
\text { al., } 2004^{(20)}\end{array}$ & Venezuela & Transversal & $\begin{array}{c}\text { Crianças de } \\
28-84 \text { meses } \\
(202)\end{array}$ & $\begin{array}{c}\text { Ferro } \\
\text { Vitamina A }\end{array}$ & $\begin{array}{c}\text { Z-escore } \\
\text { de } E / I \\
\text { Z-escore } \\
\text { de } \mathrm{P} / \mathrm{I}\end{array}$ & $\begin{array}{l}\text { - E/I<2Z: } 14,4 \% \\
\text { - P/I<2Z: } 9,4 \% \\
\text { - Anemia: } 38,1 \% \\
\text { - Deficiência de vitamina A: } \\
21,8 \% \\
\text { - Coexistência dos déficits E/I e } \\
\text { P/I com a anemia em 5,94\% e } \\
\text { 2,97\% das crianças, } \\
\text { respectivamente } \\
\text { - Coexistência dos déficits E/I e } \\
\text { P/I com a deficiência de } \\
\text { vitamina A em 2,97\% e 1,48\% } \\
\text { das crianças, respectivamente } \\
\text { - Coexistência da deficiência de } \\
\text { vitamina A com a anemia em } \\
\text { 7,92\% das crianças }\end{array}$ & 09 \\
\hline $\begin{array}{l}\text { Oelofse et al., } \\
2002^{(21)}\end{array}$ & $\begin{array}{c}\text { África do } \\
\text { Sul }\end{array}$ & Transversal & $\begin{array}{c}\text { Crianças de } \\
\text { 6-12 meses } \\
(120)\end{array}$ & $\begin{array}{c}\text { Ferro } \\
\text { Vitamina A } \\
\text { Zinco }\end{array}$ & $\begin{array}{c}\text { Z-escore } \\
\text { de E/I } \\
\text { Z-escore } \\
\text { de } P / E \\
\text { Z-escore } \\
\text { de } P / I\end{array}$ & $\begin{array}{l}\text { - E/I }<2 Z: 12,5 \% \\
\text { - P/E<2Z: } 5,0 \% \\
\text { - P/I<2Z: } 0,0 \% \\
\text { - Anemia: } 74,1 \% \\
\text { - Deficiência de vitamina A: } \\
\text { 13,5\% } \\
\text { - Deficiência de zinco: } 33,7 \% \\
\text { - Coexistência da deficiência de } \\
\text { dois micronutrientes maior } \\
\text { entre o zinco e o ferro e menor } \\
\text { entre a vitamina A e o zinco } \\
\text { - Coexistência da deficiência de } \\
\text { dois ou mais micronutrientes } \\
\text { variou de } 6,0 \% \text { (crianças } \\
\text { brancas) a } 43,0 \% \text { (crianças } \\
\text { negras) } \\
\text { - Coexistência da deficiência de } \\
\text { vitamina A, da anemia e da } \\
\text { deficiência de zinco de } 4,0 \% \text { nas } \\
\text { crianças negras }\end{array}$ & 06 \\
\hline
\end{tabular}




\begin{tabular}{|c|c|c|c|c|c|c|c|c|}
\hline \multirow{4}{*}{ 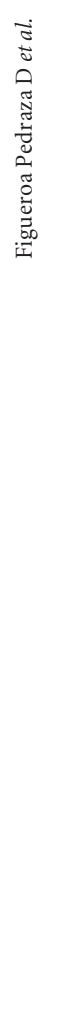 } & Tabela 2. cont & inuação & 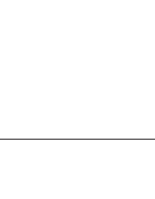 & 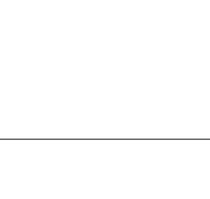 & 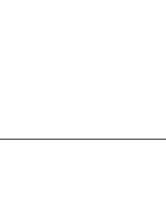 & 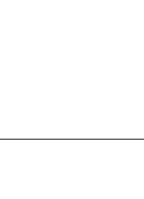 & & \\
\hline & \multirow[t]{2}{*}{ Autor, ano } & \multirow[t]{2}{*}{ País } & \multirow{2}{*}{$\begin{array}{l}\text { Tipo de } \\
\text { estudo }\end{array}$} & \multirow{2}{*}{$\begin{array}{l}\text { População } \\
\text { (amostra) }\end{array}$} & \multicolumn{2}{|c|}{ Dados de interesse } & \multirow[t]{2}{*}{ Principais resultados ${ }^{*}$} & \multirow[t]{2}{*}{ Escore $^{* *}$} \\
\hline & & & & & Exposição & Desfecho & & \\
\hline & $\begin{array}{l}\text { Dijkhuizen } \\
\text { et al., } 2001^{(22)}\end{array}$ & Indonésia & Transversal & $\begin{array}{c}\text { Crianças de } \\
2,4-10,5 \text { meses } \\
(197)\end{array}$ & $\begin{array}{c}\text { Ferro } \\
\text { Vitamina A } \\
\text { Zinco }\end{array}$ & $\begin{array}{c}\text { Z-escore } \\
\text { de E/I } \\
\text { Z-escore } \\
\text { de P/E }\end{array}$ & $\begin{array}{l}\text { - E/I<2Z: } 7,0 \% \\
\text { - P/E<2Z: } 0,6 \% \\
\text { - Anemia: } 57,0 \% \\
\text { - Deficiência de vitamina A: } \\
54,0 \% \\
\text { - Deficiência de zinco: } \\
\text { 17,0\% } \\
\text { - Ausência de associação } \\
\text { estatística entre as } \\
\text { concentrações de } \\
\text { micronutrientes e os índices } \\
\text { antropométricos } \\
\text { - A deficiência de vitamina } \\
\text { A aumentou o risco das } \\
\text { deficiências de zinco e de } \\
\text { anemia em } 2,9 \text { e } 2,5 \text { vezes, } \\
\text { respectivamente }\end{array}$ & 13 \\
\hline
\end{tabular}

A faixa etária das crianças diferiu entre os estudos. Cinco estudos ${ }^{13,15,18,20,24}$ incluíram crianças maiores de cinco anos, dois ${ }^{19,22}$ incluíram crianças menores de seis meses e outros dois foram realizados com crianças de até 12 meses de idade $^{21,22}$. Albalak et al. ${ }^{23}$ estudaram crianças de duas faixas etárias diferentes: 12-35,9 meses e 3659,9 meses. Assim mesmo, Hautvast et al. ${ }^{25}$ observaram crianças de 6-9 meses e 14-20 meses.

Os estudos tipo coorte, realizados na Zâmbia $^{25}$ e na França ${ }^{26}$, indicaram associação estatística entre a deficiência de ferro e a baixa estatura. $\mathrm{O}$ primeiro avaliou a $\mathrm{E} / \mathrm{I}$ e os micronutrientes zinco (zinco sérico), ferro (hemoglobina) e vitamina A (retinol sérico) em duas coortes de crianças residentes em uma localidade rural com altas prevalências de desnutrição crônica. O segundo incluiu como variáveis de interesse o ganho de estatura, o zinco (zinco sérico) e o ferro (ferritina sérica), pesquisando crianças menores de três anos, saudáveis, atendidas em um hospital universitário. Estes estudos também apontaram como resultado importante a associação entre os níveis de hemoglobina e retinol sérico, no caso do primeiro estudo, e a ausência de associação entre os níveis séricos de zinco e de ferritina, no segundo caso.

Considerando os estudos transversais, todos os artigos utilizaram o índice E/I como parâme- tro de desfecho. Cem por cento dos artigos estudaram o ferro; 78,6\%, a vitamina A e 50,0\%, o zinco. A coexistência entre o déficit de crescimento e a deficiência de vitamina A foi analisada em dois artigos, variando de $2,97 \%{ }^{20}$ a $20,5 \%{ }^{23}$. A coexistência entre anemia e baixa estatura foi analisada em três artigos ${ }^{13,20,23}$, variando de $5,94 \%{ }^{20}$ a $24,1 \%{ }^{13}$. A associação estatística entre as deficiências de micronutrientes e o déficit de estatura foi verificada em 10 estudos ${ }^{13-19,22-24}$. Os resultados mostram que nenhum dos artigos apontou a associação para o caso do zinco, dois apontaram a associação para o caso da vitamina $\mathrm{A}^{23,24}$, e três apontaram a associação para o caso do ferro ${ }^{15,18,23}$. A associação estatística entre as concentrações do indicador bioquímico de deficiência de vitamina A e de hemoglobina foi apontada em quatro estudos ${ }^{14,15,23,24}$.

A associação entre o estado nutricional de ferro e o crescimento linear foi constatada por del Real et al. ${ }^{15}$ ao analisar crianças entre 4-7 anos da zona suburbana de Valencia, Venezuela, caracterizada por altos níveis de pobreza; por de Abreu et al. ${ }^{18}$ ao estudar crianças menores de 10 anos de uma comunidade urbana de Caracas, Venezuela, atendidas em um centro de atenção nutricional; e por Albalak et al. ${ }^{23}$ ao avaliar 1243 crianças hondurenhas de 1 a 5 anos, encontrando-se associação tanto em crianças de 12-35,9 meses quanto 
Tabela 2. continuação

\begin{tabular}{|c|c|c|c|c|c|c|c|}
\hline \multirow[t]{2}{*}{ Autor, ano } & \multirow[t]{2}{*}{ País } & \multirow{2}{*}{$\begin{array}{l}\text { Tipo de } \\
\text { estudo }\end{array}$} & \multirow{2}{*}{$\begin{array}{c}\text { População } \\
\text { (amostra) }\end{array}$} & \multicolumn{2}{|c|}{ Dados de interesse } & \multirow[t]{2}{*}{ Principais resultados ${ }^{*}$} & \multirow[t]{2}{*}{ Escore $^{* *}$} \\
\hline & & & & Exposição & Desfecho & & \\
\hline $\begin{array}{l}\text { Albalak et } \\
\text { al., } 2000^{(23)}\end{array}$ & Honduras & Transversal & $\begin{array}{c}\text { Crianças de } \\
12-35,9 \text { meses } \\
(633)\end{array}$ & $\begin{array}{c}\text { Ferro } \\
\text { Vitamina A }\end{array}$ & $\begin{array}{c}\text { Z-escore } \\
\text { de E/I }\end{array}$ & $\begin{array}{l}\text { - E/I }<2 \mathrm{Z}: 35 \% \text { (crianças de } 12- \\
35,9 \text { meses) e } 38 \% \text { (crianças de } \\
36-59,9 \text { meses) }\end{array}$ & 7 \\
\hline
\end{tabular}

- Anemia: 40\% (crianças de 1235,9 meses) e $18 \%$ (crianças de 36-59,9 meses)

- Deficiência de vitamina A: 48\% (crianças de 12-35,9 meses) e $42 \%$ (crianças de 36 59,9 meses)

- Associação estatística entre as concentrações de retinol sérico e o Z-escore E/I nas crianças de 12-35,9 meses e de 36-59,9 meses

- Associação estatística entre as concentrações de hemoglobina e o Z-escore E/I nas crianças de 36-59,9 meses

- Associação estatística entre as concentrações de hemoglobina e de retinol sérico nas crianças de 12-35,9 meses e de 36-59,9 meses

- Coexistência da deficiência de vitamina $\mathrm{A}$, anemia e déficit de estatura em $8,4 \%$ das crianças de $12-35,9$ meses e em $4,8 \%$ das crianças de 36-59,9 meses - Coexistência da deficiência de vitamina A com o déficit de estatura em 19,4\% das crianças de $12-35,9$ meses e em 20,5\% das crianças de 36-59,9 meses - Coexistência da anemia com o déficit de estatura em 15,2\% das crianças de 12-35,9 meses e em 8,2\% das crianças de 36-59,9 meses

- Coexistência da deficiência de vitamina A com a anemia em $21,6 \%$ das crianças de 12-35,9 meses e em 9,3\% das crianças de 36-59,9 meses

em crianças de 36-59,9 meses $(n=610)$. Neste último artigo, observaram-se altas prevalências de déficit de estatura associadas, também, à deficiência de vitamina A. A associação entre os indicadores de baixa estatura e de deficiência de vita- mina A resultou igualmente significativa na avaliação de 1678 crianças, também hondurenhas, de 12-71 meses $^{24}$.

A ausência de associação entre as deficiências de ferro, vitamina A ou zinco e a baixa estatura 
Tabela 2. continuação

\begin{tabular}{|c|c|c|c|c|c|c|c|}
\hline \multirow[t]{2}{*}{ Autor, ano } & \multirow[t]{2}{*}{ País } & \multirow{2}{*}{$\begin{array}{l}\text { Tipo de } \\
\text { estudo }\end{array}$} & \multirow{2}{*}{$\begin{array}{c}\text { População } \\
\text { (amostra) }\end{array}$} & \multicolumn{2}{|c|}{ Dados de interesse } & \multirow[t]{2}{*}{ Principais resultados ${ }^{*}$} & \multirow[t]{2}{*}{ Escore $^{* *}$} \\
\hline & & & & Exposiçãa & Desfecho & & \\
\hline $\begin{array}{l}\text { Nestel } \\
\text { et al., } \\
1999^{(24)}\end{array}$ & Honduras & Transversal & $\begin{array}{c}\text { Crianças de } \\
\text { 36-59,9 meses } \\
(610)\end{array}$ & $\begin{array}{c}\text { Ferro } \\
\text { Vitamina A }\end{array}$ & $\begin{array}{c}\text { Z-escore } \\
\text { de E/I } \\
\text { Z-escore } \\
\text { de } P / I\end{array}$ & $\begin{array}{l}\text { - E/I<2Z (dado não disponível) } \\
\text { - P/I<2Z (dado não disponível) } \\
\text { - Anemia: } 30 \% \\
\text { - Deficiência de vitamina A: } \\
14 \% \\
\text { - Associação estatística entre as } \\
\text { concentrações de retinol } \\
\text { plasmático e os indicadores } \\
\text { antropométricos } \\
\text { - Associação estatística } \\
\text { significativa entre as } \\
\text { concentrações de hemoglobina } \\
\text { e o Z-escore de P/I } \\
\text { - Associação estatística } \\
\text { significativa entre as } \\
\text { concentrações de retinol } \\
\text { plasmático e de hemoglobina }\end{array}$ & 10 \\
\hline $\begin{array}{l}\text { Hautvast } \\
\text { et al., } \\
2000^{(25)}\end{array}$ & Zâmbia & $\begin{array}{c}\text { Coorte } \\
(21 \text { meses })\end{array}$ & $\begin{array}{c}\text { Crianças de } \\
\text { 12-71 meses } \\
(1678) \\
\text { Crianças de } \\
6-9 \text { meses }(108) \\
\text { Crianças de } \\
14-20 \text { meses } \\
(102)\end{array}$ & $\begin{array}{c}\text { Ferro } \\
\text { Vitamina A } \\
\text { Zinco }\end{array}$ & $\begin{array}{l}\text { Z-escore } \\
\text { de E/I }\end{array}$ & $\begin{array}{l}\text { - E/I<2Z: } 36,0-79,0 \% \\
\text { - Anemia: } 78,0-99,0 \% \\
\text { - Deficiência de vitamina A } \\
\text { (dado não disponível) } \\
\text { - Deficiência de zinco (dado } \\
\text { não disponível) } \\
\text { - Associação estatística entre as } \\
\text { concentrações de hemoglobina } \\
\text { e os Z-escore de E/I } \\
\text { - Ausência de associação } \\
\text { estatística entre as } \\
\text { concentrações de retinol sérico } \\
\text { e os Z-escore de E/I } \\
\text { - Ausência de associação } \\
\text { estatística entre as } \\
\text { concentrações de zinco sérico e } \\
\text { os Z-escore de E/I } \\
\text { - Associação estatística entre a } \\
\text { estatura materna e os Z-escore } \\
\text { de E/I } \\
\text { - Ausência de associação } \\
\text { estatística entre a situação } \\
\text { socioeconômica e os Z-escore } \\
\text { de E/I } \\
\text { - A estatura das crianças } \\
\text { mostrou uma forte relação } \\
\text { com a estatura em idades } \\
\text { posteriores } \\
\text { - Associação estatística entre as } \\
\text { concentrações de hemoglobina } \\
\text { e de retinol sérico }\end{array}$ & 10 \\
\hline
\end{tabular}


Tabela 2. continuação

\begin{tabular}{|c|c|c|c|c|c|c|c|}
\hline \multirow[t]{2}{*}{ Autor, ano } & \multirow[t]{2}{*}{ País } & \multirow{2}{*}{$\begin{array}{l}\text { Tipo de } \\
\text { estudo }\end{array}$} & \multirow{2}{*}{$\begin{array}{c}\text { População } \\
\text { (amostra) }\end{array}$} & \multicolumn{2}{|c|}{ Dados de interesse } & \multirow[t]{2}{*}{ Principais resultados ${ }^{*}$} & \multirow[t]{2}{*}{ Escore $^{* *}$} \\
\hline & & & & Exposição & Desfecho & & \\
\hline $\begin{array}{l}\text { Bouglé et al., } \\
2000^{(26)}\end{array}$ & França & $\begin{array}{c}\text { Coorte } \\
(24 \pm 6 \\
\text { semanas })\end{array}$ & $\begin{array}{c}\text { Crianças } \\
<3 \text { anos } \\
(66)\end{array}$ & $\begin{array}{l}\text { Ferro } \\
\text { Zinco }\end{array}$ & $\begin{array}{c}\text { Peso } \\
\text { Estatura }\end{array}$ & $\begin{array}{l}\text { - Ganho de estatura: } 0,7 \pm 1,4 \mathrm{~cm} / \\
\text { semana } \\
\text { - Ganho de peso: } 115 \pm 152 \mathrm{~g} / \mathrm{semana} \\
\text { - Baixos níveis de ferritina: } 10,0 \% \\
\text { - Deficiência de zinco: } 20,0 \% \\
\text { - Associação estatística entre as } \\
\text { concentrações de ferritina sérica e o } \\
\text { ganho de estatura, considerando o } \\
\text { efeito da idade } \\
\text { - Ausência de associação estatística } \\
\text { entre as concentrações de zinco no } \\
\text { soro e ganho de estatura } \\
\text { - Associação estatística entre as } \\
\text { concentrações de ferritina sérica e o } \\
\text { ganho de peso } \\
\text { - Ausência de associação estatística } \\
\text { entre as concentrações de zinco no } \\
\text { soro e o ganho de peso } \\
\text { - Ausência de associação estatística } \\
\text { entre as concentrações de } \\
\text { hemoglobina e de zinco sérico }\end{array}$ & 11 \\
\hline
\end{tabular}

"Os casos em que não é referido o resultado sobre a associação estatística entre um determinado micronutriente e o déficit de estatura deve-se à indisponibilidade do dado no artigo. ${ }^{* *}$ Escore: escore de qualidade de acordo com Downs \& Black $^{10}$.

foi observada nas crianças dos trabalhos desenvolvidos por Chen et al. ${ }^{13}$; Lander et al. ${ }^{14}$; Poveda et al. ${ }^{16}$; Borges et al. ${ }^{17}$; Mamiro et al. ${ }^{19}$; e Dijkhuizen et al. ${ }^{22}$. Chen et al. ${ }^{13}$, em um estudo no subúrbio da cidade de Chongquing, China, envolvendo crianças de 2-7 anos e Borges et al. ${ }^{17}$, ao estudar uma comunidade de baixa renda no município de Duque de Caxias, Rio de Janeiro, envolvendo crianças de 1-5 anos, encontraram as menores prevalências de déficit de estatura entre os estudos da revisão. Por sua vez, prevalências de déficit de estatura indicativas de problema grave ( $\geq 25 \%$ ), além das mostradas por Albalak et al. ${ }^{23}$, também foram achadas por Mamiro et al. ${ }^{19}$ ao estudar crianças entre 3-23 meses da zona rural de um distrito da Tanzânia, reconhecido por elevados níveis de desnutrição e de anemia ferropriva na infância.

Lander et al. ${ }^{14}$ trabalharam em quatro distritos de Ulaanbaatar, capital da Mongólia, e em quatro capitais provincianas do país, com crianças entre 6-36 meses de idade. Os resultados mostraram altas prevalências de anemia, de deficiência de vitamina A, de deficiência de zinco e de déficit de estatura, com marcadas diferenças regio- nais. Poveda et al., $2007^{16}$ pesquisaram crianças pré-escolares assistidas em instituições públicas ou privadas de áreas urbana e rural do município de Funza, Colômbia. Os resultados revelaram alta prevalência de anemia, baixa prevalência de vitamina $\mathrm{A}$ e $\mathrm{E} / \mathrm{I}<2 \mathrm{Z}$ de 9,7\%. Dijkhuizen et al. ${ }^{22}$ investigaram crianças menores de 12 meses em duas aldeias rurais do distrito de Bogor, Indonésia. Os resultados indicaram altas prevalências de deficiências de ferro, vitamina A e zinco, com prevalência de déficit de estatura bem menor.

O escore metodológico foi, em média, de 9,29 pontos ( $\mathrm{dp}=2,09$ pontos). Trinta e seis por cento dos artigos tiveram pontuação igual ou inferior a oito ${ }^{15,16,18,21,23}$, sendo que a menor pontuação obtida foi seis ${ }^{21}$ e a maior foi $13^{22}$. As principais limitações dos estudos, segundo os critérios de Downs e Black ${ }^{10}$, estiveram na qualidade da descrição e discussão dos principais fatores de confusão e na comprovação da representatividade da amostra estudada em relação à população de estudo. O controle adequado das variáveis de confundimento na análise de associações foi notado em sete estudos ${ }^{13,14,19,22,24-26}$, obtendo os maiores escores de qualidade. 


\section{Discussão}

Apesar de sua diminuição, a desnutrição crônica continua sendo o principal problema de saúde pública na maioria dos países em desenvolvimento. $\mathrm{Na}$ atualidade, em muitas regiões do mundo, em especial na África e na Ásia, é observada uma desaceleração ou estagnação na queda dessa prevalência ${ }^{27}$. Nos países da América Latina observam-se, principalmente, tendência de declínio da desnutrição na infância. Entretanto, essa melhora caracteriza-se, a exemplo do Brasil, por diferenças regionais que ainda persistem e iniquidades que afetam as populações infantis mais pobres, residentes nas áreas rurais ou na periferia das cidades mais ricas ${ }^{28,29}$. Assim, em países economicamente desprivilegiados, o déficit de estatura pode ser utilizado como indicador de iniquidade socioeconômica ${ }^{30}$. Localidades rurais de Tanzânia ${ }^{19}$ e Zâmbia, África ${ }^{25}$, foram as que apresentaram maiores prevalências de baixa estatura no contexto deste trabalho.

$\mathrm{O}$ crescimento linear resulta da interação entre a carga genética e os fatores do meio ambiente, os quais permitirão a maior ou menor expressão do potencial genético, destacando a alimentação, a saúde, a higiene, a habitação e os cuidados gerais com a criança, que atuam acelerando ou retardando esse processo ${ }^{31}$. Infecções e consumo alimentar inadequado são causas bem estabelecidas de baixa estatura, porém, o possível papel da deficiência de micronutrientes específicos na etiologia do déficit de crescimento tem despertado atenção recentemente. Assim, o completo potencial genético de uma criança para o crescimento físico pode ser comprometido devido a deficiências subclínicas de micronutrientes. Esta deficiência pode associar-se à desnutrição proteico-calórica ou depender da absorção insuficiente dos mesmos ${ }^{32}$.

A associação entre o estado nutricional de ferro e o crescimento linear foi encontrada em 30\% (3 de 10) dos estudos transversais incluídos na presente revisão que realizaram esta análise. A associação em relação à vitamina $\mathrm{A}$ e ao zinco foi encontrada em $25 \%$ ( 2 de 8 ) e $0 \%$ (0 de 5) dos estudos transversais com resultados dessas análises, respectivamente. Por outro lado, os dois estudos de coorte também mostraram associação entre o ferro e a estatura das crianças, assim como a ausência de associação nas análises que realizaram em relação à vitamina $\mathrm{A}$ e ao zinco. Assim, somado aos anteriores resultados o da associação entre o estado nutricional de ferro e o crescimento linear em três estudos com adequa- do controle das variáveis de confundimento, evidencia-se maior consistência para a associação entre a anemia e o déficit de estatura.

A ausência de associação estatística entre as deficiências de micronutrientes e o déficit de estatura não invalida a associação epidemiológica, pois o risco indica apenas o aumento na probabilidade de déficit de estatura. Diferenças entre as populações podem implicar em resultados distintos na estimativa da participação de um fator de risco em uma doença. É isso o que legitima a necessidade de avaliar os mesmos fatores em populações diferentes ${ }^{33}$ (nota-se que a associação estatística dos estudos seccionais ficou centrada na Venezuela e Honduras). Entretanto, a consistência da associação que deve ser esperada, para ilustrar a associação entre as deficiências de micronutrientes e o déficit no crescimento linear, precisa de resultados adicionais aos obtidos no presente estudo.

Além disso, o estudo da associação entre as deficiências de micronutrientes e o déficit de estatura apresenta problemas metodológicos e analíticos relacionados com a plausibilidade e especificidade da associação: i) o impacto dos micronutrientes não pode ser separado daquele ocasionado pela quantidade ou qualidade de energia e/ou proteína, ii) o impacto de uns micronutrientes não pode ser separado daquele ocasionado por outros (devido à deficiência simultânea de vários micronutrientes), iii) o impacto de um micronutriente está relacionado ao seu nível de deficiência, de biodisponibilidade e à magnitude do déficit de estatura (diferenças que prescindem de suficientes estudos para serem observadas).

$\mathrm{Na}$ conjuntura da última questão, ressalta a etiologia multifatorial do déficit no crescimento linear ${ }^{31} \mathrm{em}$ um contexto que predomina a prevalência generalizada de anemia ${ }^{34}$, sustentando a possibilidade de a anemia ferropriva contribuir com o déficit de estatura. Por sua vez, como a influência da vitamina A no crescimento infantil está relacionada à proteção de doenças infeccio$\operatorname{sas}^{35}$, sua deficiência severa ser de pouca magnitude e apresentar características endêmicas ${ }^{36,37}$, a contribuição da deficiência de vitamina A nas prevalências de baixa estatura deve ser mínima. Reconhecido o efeito direto do zinco no crescimento linear, com prejuízos mesmo nos casos de deficiência leve $e^{35}$, a deficiência de zinco deve contribuir de forma importante com o déficit de estatura em áreas onde apresenta prevalências elevadas, sugerindo a concorrência com a anemia ferropriva.

Uma vez que o déficit de estatura é uma condição que ainda apresenta prevalências impor- 
tantes, porém não expressivas como das deficiências de micronutrientes, cogita-se na possibilidade de associação mais consistente se fossem consideradas as formas leves de déficit de estatura. Talvez, nesta última situação, o zinco poderia expressar mais fortemente sua importância no crescimento infantil. Nesse argumento, ressaltam-se as limitações geradas pelo menor conhecimento sobre a prevalência, distribuição e etiologia da deficiência de zinco ${ }^{38}$.

Considera-se ainda a importância da complementação dos resultados de estudos transversais com longitudinais. Esses últimos podem oferecer subsídios para uma compreensão mais adequada da situação de saúde e nutrição, e conduzir a um melhor entendimento de um processo dinâmico, contínuo e de rápidas mudanças, como o crescimento e o desenvolvimento infantil. Desenvolver abordagens comparativas envolvendo a coleta de dados em diversas populações simultaneamente, de modo a incrementar a comparabilidade dos achados, também é destacado ${ }^{39,40}$.

\section{Conclusões}

Fatores de caráter biológico e a magnitude das respectivas deficiências dificultam o estabelecimento da associação entre o déficit de estatura e as carências nutricionais de ferro, vitamina A e zinco. Os resultados apresentados permitem a composição de um panorama preliminar da associação da anemia e o déficit de estatura. As elevadas prevalências de déficit de estatura que ainda persistem nos países em desenvolvimento, a carga de morbidade que se associa a esse evento, assim como a redução das taxas do déficit de estatura em crianças através da suplementação efetiva com micronutrientes, explicam a importância de tais conhecimentos.

\section{Considerações finais}

A comparação dos resultados de diferentes estudos está atrelada à padronização metodológica. Nesse contexto, os artigos incluídos na presente revisão, apesar da adoção de critérios de inclusão e exclusão muito bem definidos que representa maiores possibilidades de comparação, apresentaram diversidade de formas na apresentação dos resultados. Destacam-se as diferenças nos agrupamentos etários, nas populações-referência, nos pontos de corte utilizados e no tratamento do efeito da infecção subclínica nas concentrações de hemoglobina, de retinol sérico e zinco sérico. A apresentação dos resultados antropométricos das populações infantis deve incluir, como forma de padronização mínima, a publicação de tabelas com parâmetros quantitativos básicos (ex.: média e desvio-padrão das várias medidas), segundo sexo e grupos de idade.

Pesquisas são necessárias para padronizar, monitorar e estabelecer normas que ajudem na obtenção de resultados adequados e comparáveis, entre elas:

- estabelecer critérios uniformes para o desenho de inquéritos nutricionais (ex.: frequência, tamanho da amostra, técnicas de medição, apresentação dos dados por idade, etc.);

- desenvolver e padronizar os métodos para a medição do estado nutricional (o desenvolvimento de técnicas de avaliação bioquímica, de baixo custo e que precisem de quantidades mínimas de sangue, são essenciais);

- revisar os indicadores e pontos de corte utilizados para avaliar a gravidade do déficit de estatura e as deficiências de ferro, vitamina A e zinco, considerando, inclusive, a influência da infecção subclínica.

\section{Colaboradores}

Figueroa Pedraza D participou da elaboração do projeto, concepção do artigo, revisão bibliográfica, análise e interpretação dos dados e redação final do artigo. Rocha ACD e Sales MC participaram da revisão bibliográfica, análise e interpretação dos dados e redação final do artigo. 


\section{Referências}

1. Black RE. Micronutrients in pregnancy. Br J Nutr 2001; 85(Supl. 2):193-197.

2. Rosado JL. Separate and joint effects of micronutrient deficiencies on linear growth. J Nutr 1999; 129(Supl. 2):531-533.

3. Valente SL de S, Thiapó AP, Souza GG de, Saunders C, Ramalho A. Micronutrientes na gestação e lactação. Rev Bras Saúde Matern Infant 2007; 7(3):237244.

4. Oliveira JM, Michelazzo FB, Stefanello J, Rondó PHC. Influence of iron on vitamin A nutritional status. Nutr Rev 2008; 66(3):141-147.

5. Christian P, West KP. Interactions between zinc and vitamin A: an update. Am J Clin Nutr 1998; 68(Supl.):435-441.

6. Gibson RS, Hotz C. Nutritional causes of linear growth faltering in infants during the complementary feeding period. In: Martorell R, Haschke F, editors. Nutrition and Growth. Nestle Nutrition Workshop Series Pediatric Program. Vol 47. Philadelphia: Lippincott, Williams \& Wilkins; 2001. p.159192.

7. Neufeld L, Hotz C. Para el Proyecto Challenges for Childhood Health and Nutrition Research in Latin America: addressing the 90/10 gap. Restricción en el crecimiento $y$ deficiencia de micronutrientes. Bangladesh: Child Health and Nutrition Research Initiative; 2003.

8. Rivera Dommarco J, Hotz C, Gonzalez de Cossío T, Neufeld L, Garcia Guerra A. The effect of micronutrient deficiencies on child growth: a review of results from community-based supplementation trials. J Nutr 2003; 133(11 Supl.2):4010-4020.

9. Bhandari N, Bahl R, Taneja S. Effect of micronutrient supplementation on linear growth of children. Br J Nutr 2001; 85(Supl.2):S131-137.

10. Downs HS, Black N. The feasibility of creating a checklist for the assessment of the methodological quality both of randomised and non-randomised studies of health care interventions. J Epidemiol Community Health 1998; 52(6):377-384.

11. Sanderson S, Tatt ID, Higgins JPT. Tools for assessing quality and susceptibility to bias in observational studies in epidemiology: a systematic review and annotated bibliography. Int J Epidemiol 2007; 36(3):666-676.
12. Vandenbroucke JP, Von Elm E, Altman DG, Gotzsche PC, Mulrow CD, Pocock SJ, Poole C, Schlesselman JJ, Egger M, STROBE Initiative. Strengthening the reporting of observational studies in epidemiology (STROBE): explanation and elaboration. Epidemiology 2007; 18(6):805-835.

13. Chen K, Zhang X, Li TY, Chen L, Qu P, Liu YX. Coassessment of iron, vitamin $\mathrm{A}$ and growth status to investigate anemia in preschool children in suburb Chongqing, China. World J Pediatr 2009; 5(4):275281.

14. Lander RL, Enkhjargal T, Batjargal J, Bailey KB, Diouf S, Green TJ, Skeaff CM, Gibson RS. Multiple micronutrient deficiencies persist during early childhood in Mongolia. Asia Pac J Clin Nutr 2008; 17(3):429-440.

15. Del Real SI, Sánchez Jaeger A, Barón MA, Díaz N, Solano L, Velásquez E, López J. Estado nutricional en niños preescolares que asisten a un jardín de infancia público en Valencia, Venezuela. Arch Latinoam Nutr 2007; 57(3):248-254.

16. Poveda E, Cuartas A, Guarín S, Forero Y, Villarreal E. Estado de los micronutrientes hierro y vitamina A, factores de riesgo para las deficiencias y valoración antropométrica en niños preescolares del municipio de Funza, Colombia. Biomédica 2007; 27(1):76-93.

17. Borges CVD, Veiga APB, Barroso GS, Jesus EFO, Serpa RFB, Moreira, S, Salles-Costa R. Associação entre concentrações séricas de minerais, índices antropométricos e ocorrência de diarréia entre crianças de baixa renda da região metropolitana do Rio de Janeiro. Rev Nutr 2007; 20(2):159-169.

18. De Abreu J, Borno S, Montilla M, Dini E. Anemia y deficiencia de vitamina $\mathrm{A}$ en niños evaluados en un centro de atención nutricional de Caracas. Arch Latinoam Nutr 2005; 55(3):226-234.

19. Mamiro PS, Kolsteren P, Roberfroid D, Tatala S, Opsomer AS, Van Camp JH. Feeding practices and factors contributing to wasting, stunting, and irondeficiency anaemia among 3-23-month old children in Kilosa district, rural Tanzania. J Health Popul Nutr 2005; 23(3):222-230.

20. Castejon HV, Ortega P, Amaya D, Gomez G, Leal J, Castejon OJ. Co-existence of anemia, vitamin A deficiency and growth retardation among children 24-84 months old in Maracaibo, Venezuela. Nutr Neurosci 2004; 7(2):113-119. 
21. Oelofse A, Van Raaij JM, Benadé AJ, Dhansay MA, Tolboom JJ, Hautvast JG. Disadvantaged black and coloured infants in two urban communities in the Western Cape, South Africa differ in micronutrient status. Public Health Nutrition 2002; 5(2):289-294.

22. Dijkhuizen MA, Wieringa FT, West CE, Muherdiyantiningsih, Muhilal. Concurrent micronutrient deficiencies in lactating mothers and their infants in Indonesia. Am J Clin Nutr 2001; 73(4):786-791.

23. Albalak R, Ramakrishnan U, Stein AD, Haar FV, Haber MJ, Schroeder D, Martorell R. Co-Occurrence of Nutrition Problems in Honduran Children. J Nutr 2000; 130(9):2271-2273.

24. Nestel P, Melara A, Rosado J, Mora JO. Vitamin A deficiency and anemia among children 12-71 months old in Honduras. Rev Panam Salud Publica 1999; 6(1):34-43.

25. Hautvast JLA, Tolboom JJM, Kafwembe EM, Musonda RM, Mwanakasale V, Staveren WAV, van 't Hof MA, Sauerwein RW, Willems JL, Monnens LA. Severe linear growth retardation in rural Zambian children: the influence of biological variables. $A m$ J Clin Nutr 2000; 71(2):550-559.

26. Bouglé D, Laroche D, Bureau F. Zinc and iron status and growth in healthy infants. Eur J Clin Nutr 2000; 54(10):764-767.

27. de Onis M, Blössner M, Borghi E. Prevalence and trends of stunting among pre-school children, 19902020. Public Health Nutrition 2012; 15(1):142-148.

28. Victora CG, Aquino EML, Leal MC, Monteiro CA, Barros FC, Szwarcwald CL. Saúde de mães e crianças no Brasil: progressos e desafios. Lancet [periódico na Internet] 2011 maio [acessado $2011 \mathrm{fev} \mathrm{10];}$ 6736(11):32-46. Disponível em: http://download. thelancet.com/flatcontentassets/pdfs/brazil/ brazilpor2.pdf

29. Coutinho JG, Gentil PC, Toral N. A desnutrição e obesidade no Brasil: o enfrentamento com base na agenda única da nutrição. Cad Saude Publica 2008; 24(Supl.2):S332-340.

30. Oliveira FCC, Cotta RMM, Ribeiro AQ, Sant'Ana LFR, Priore SE, Franceschini SCC. Estado nutricional e fatores determinantes do déficit estatural em crianças cadastradas no Programa Bolsa Família. Epidemiol Serv Saúde 2011; 20(1):7-18.

31. Romani SAM, Lira PIC. Fatores determinantes do crescimento infantil. Rev Bras Saúde Matern Infant 2004; 4(1):15-23
32. Bueno AL, Czepielewski MA. Micronutrientes envolvidos no crescimento. Rev HCPA 2007; 27(3):4756.

33. Alvarez H, Alvarez A. Investigación y epidemiología. 1ª Edição. Santa Fe de Bogotá: Ecoe Ediciones; 1998.

34. Batista Filho M, Souza AI, Bresani CC. Anemia como problema de saúde pública: uma realidade atual. Cien Saude Colet 2008; 13(6):1917-1922.

35. Figueroa Pedraza D, Queiroz D. Micronutrientes no crescimento e desenvolvimento infantil. Rev Bras Crescimento Desenvolvimento Hum 2011; 21(1):155170.

36. Ramalho RA, Flores H, Saunders C. Hipovitaminose A no Brasil: um problema de saúde pública. Rev Panam Salud Publica 2002; 12(2):117-123.

37. Souza WA, Vilas Boas OMG. A deficiência de vitamina A no Brasil: um panorama. Rev Panam Salud Publica 2002; 12(3):173-179.

38. Figueroa Pedraza D, Rocha ACD, Queiroz EO, Souza CPC. Estado nutricional relativo ao zinco de crianças que frequentam creches do estado da $\mathrm{Pa}$ raíba. Rev Nutr 2011; 24(4):539-552.

39. Lira PIC, Lima MC, Silva GAP, Romani SAM, Eickmann SH, Alessio MLM, Batista Filho M, Leger CL, Huttly SR, Ashworth A. Saúde e nutrição de crianças de áreas urbanas da Zona da Mata Meridional de Pernambuco: resultados preliminares de um estudo de coorte. Rev Bras Saúde Matern Infant 2003; 3(4):463-472.

40. Barros AJD, Santos IS, Victoria CG, Albernaz EP, Domingues MR, Timm IK, Matijasevich A, Bertoldi AD, Barros FC. Coorte de nascimentos, Pelotas, 2004: metodologia e descrição. Rev Saude Publica 2006; 40(3):402-413.

Artigo apresentado em 05/07/2012

Aprovado em 28/08/2012

Versão final apresentada em 14/09/2012 
